10. Tamura K, Stecher G, Peterson D, Filipski A, Kumar S. MEGA6: Molecular Evolutionary Genetics Analysis version 6.0. Mol Biol Evol. 2013;30:2725-9. http://dx.doi.org/10.1093/molbev/mst197

Address for correspondence: Mutien Garigliany, University of Liege, Department of Pathology, Avenue de Cureghem 6, B43, Liège B-4000 Belgium; email: mmgarigliany@uliege.be

\title{
Angiostrongylus cantonensis DNA in Cerebrospinal Fluid of Persons with Eosinophilic Meningitis, Laos
}

\section{Damien K.Y. Ming, Sayaphet Rattanavong, Tehmina Bharucha, Onanong Sengvilaipaseuth, Audrey Dubot-Pérès, Paul N. Newton, Matthew T. Robinson}

\author{
Author affiliations: Imperial College London, London, UK \\ (D.K.Y. Ming); Lao-Oxford-Mahosot Hospital Wellcome Trust \\ Research Unit, Vientiane, Laos (D.K.Y. Ming, S. Rattanavong, \\ T. Bharucha, O. Sengvilaipaseuth, A. Dubot-Pérès, P.N. Newton, \\ M.T. Robinson); University College London, London \\ (T. Bharucha); Aix-Marseille Université, Marseille, France \\ (A. Dubot-Pérès); University of Oxford, Oxford, UK \\ (P.N. Newton, M.T. Robinson)
}

DOI: https://doi.org/10.3201/eid2312.171107

Definitive identification of Angiostrongylus cantonensis parasites from clinical specimens is difficult. As a result, regional epidemiology and burden are poorly characterized. To ascertain presence of this parasite in patients in Laos with eosinophilic meningitis, we performed quantitative PCRs on 36 cerebrospinal fluid samples; 4 positive samples confirmed the parasite's presence.

$\mathrm{H}$ umans are incidental hosts of Angiostrongylus cantonensis nematodes; global distribution of these nematodes is being increasingly recognized (1). Ingestion of larvae from undercooked infected snails or food contaminated with mollusk secretions can result in the migration of $A$. cantonensis parasites through the human central nervous system (CNS) (2). The presence of the parasite and associated inflammation in the CNS can contribute to a meningoencephalitic syndrome, typified by a cerebrospinal fluid (CSF) eosinophilia constituting $\geq 10 \%$ of total CSF leukocyte count.
Formal diagnosis of angiostrongyliasis is difficult because the parasite is typically present in low numbers within the CSF (3). Serologic methods are limited by cross-reactivity with other helminths (4), and antibody-based methods may lack sensitivity, especially during acute illness (5).

Host sampling studies have identified $A$. cantonensis parasites in some Mekong region countries but not in Lao People's Democratic Republic (Laos) (6). To ascertain the presence of this parasite in patients with eosinophilic meningitis in Laos, we tested samples from a cohort of 1,065 patients suspected of having CNS infection at Mahosot Hospital, Vientiane, Laos, during 2003-2013 by Giemsa staining and identified 54 CSF samples containing $\geq 10 \%$ eosinophils. Of these, 36 samples from 35 patients were available for this study (1 patient underwent lumbar puncture twice) and were tested by conventional and quantitative PCR (cPCR and qPCR). From the same cohort, we also performed qPCR testing on another $50 \mathrm{CSF}$ samples with $1 \%-9 \%$ eosinophils.

DNA was extracted from $200 \mu \mathrm{L}$ of CSF by using a QIAamp DNA Mini Kit (QIAGEN, Hilden, Germany) and eluted in $30 \mu \mathrm{L}$ buffer. We ran the extract in parallel with positive samples (A. cantonensis DNA from experimentally infected rats, University of Sydney, Sydney, Australia) and negative controls by using published cPCR (7) and qPCR (8) protocols. We used Platinum PCR SuperMix (Thermo Fisher, Waltham, MA, USA) and performed assays on a Bio-Rad CFX96 (Bio-Rad, Watford, UK).

Among patients with CSF eosinophilia $\geq 10 \%$, male patients predominated, although sex of patients did not differ significantly among patients with or without CSF eosinophilia (Table). Of the $36 \mathrm{CSF}$ samples that contained $\geq 10 \%$ eosinophils, all were negative by cPCR, but $4(11.1 \%)$ were positive for $A$. cantonensis DNA by qPCR; median quantification cycle was 35.9 (range 34.1-37.4). Sensitivity of qPCR was apparently higher than that of cPCR. The median duration of illness for patients with positive qPCR was 4 (range 0-10) days. Of 3 patients with a positive qPCR, 2 reported that they had eaten raw snails in the previous month.

Results from 2 CSF samples obtained from the same patient and tested by qPCR were discordant; CSF obtained after 7 days of illness was negative for $A$. cantonensis DNA, but a sample obtained on day 13 was positive (quantification cycle 34.1). This finding is consistent with previous observations (8), and it is plausible that during the acute stages of infection, insufficient nucleic material is present for detection. Although lumbar puncture is invasive, a high clinical suspicion of angiostrongyliasis in the context of a negative qPCR may therefore warrant a repeated lumbar puncture. All positive samples had CSF eosinophil proportions $>40 \%$, and all samples containing a $1 \%-9 \%$ eosinophil proportion tested negative by qPCR, supporting the conventional cutoff of a CSF eosinophilia $\geq 10 \%$ in the diagnosis of CNS angiostrongyliasis. 
Table. Demographics and clinical characteristics of 35 patients with eosinophilic meningitis tested by qPCR for Angiostrongylus cantonensis parasites*

\begin{tabular}{|c|c|c|}
\hline Characteristic & Positive, $\mathrm{n}=4 \dagger$ & Negative, $n=31 \dagger$ \\
\hline Median age, y (IQR) & $25(21-42)$ & $25(20-40)$ \\
\hline \multicolumn{3}{|l|}{ Sex, no. (\%) } \\
\hline M & $4 / 4(100)$ & $19 / 31(61)$ \\
\hline $\mathrm{F}$ & 0 & $12 / 31(39)$ \\
\hline Median duration of illness, $d(I Q R)$ & $4(1-9)$ & $4(0-6.5)$ \\
\hline Median eosinophil \% of total leukocyte count (IQR) & $65(50-73)$ & $33(14-56)$ \\
\hline \multicolumn{3}{|l|}{ Diet in past mo } \\
\hline Raw fish & $3 / 3$ & $7 / 13$ \\
\hline Raw shellfish & $2 / 2$ & $3 / 11$ \\
\hline Raw snails & $2 / 3$ & $5 / 12$ \\
\hline Raw crab & $2 / 2$ & $2 / 11$ \\
\hline Fulfilled WHO criteria for meningitis at admission, no. (\%) & $3 / 4(75)$ & $9 / 28(32)$ \\
\hline Median CSF opening pressure, $\mathrm{cm} \mathrm{H}_{2} \mathrm{O}$ (IQR) & $28(22-35)$ & $27(17-40)$ \\
\hline Median CSF leukocyte count, $\times 10^{6}$ cells/L (IQR) & $583(493-1,198)$ & $125(40-580)$ \\
\hline Median CSF protein, g/L (IQR) & $0.68(0.31-1.08)$ & $0.56(0.31-1.00)$ \\
\hline Median CSF glucose, $\mathrm{mmol} / \mathrm{L}$ (IQR) & $2.7(2.4-4.2)$ & $3.1(2.4-4.9)$ \\
\hline
\end{tabular}

Our findings are consistent with those from studies demonstrating $A$. cantonensis parasites as a cause of eosinophilic meningitis in the region, although the proportion of A. cantonensis-positive cases in our cohort was lower than that from Vietnam (11.1\% vs. 67.3\%) (9). Results may be affected by factors such as geographic location, differences in healthcare access, and the contribution of other causes of eosinophilic meningitis, such as Gnathostoma spinigerum nematodes. In the absence of a reliable reference diagnostic standard, we were unable to estimate the proportion of false-negative results in this cohort or to correlate findings with other immunodiagnostic modalities. Further studies on eosinophilic meningitis from a wider geographic area and improved diagnostics would help establish the overall clinical burden of CNS angiostrongyliasis in Laos.

Diet (consumption of raw snails and food contaminated by snails) is considered a major risk factor for angiostrongyliasis, making an epidemiologic link and public health interventions a possibility. The exact host species responsible for transmission remains unclear, however, given that $A$. cantonensis parasites have apparently yet to be identified within snails in Laos.

Our identification of $A$. cantonensis DNA by qPCR of CSF samples from $4(11 \%)$ of 35 patients with eosinophilic meningitis confirms the parasite's presence in Laos. Further regional characterization, coupled with dietary surveys, will be invaluable for stratifying the risk for human transmission and guiding public health interventions.

\section{Acknowledgments}

We are thankful for the support of the directors and staff of Mahosot Hospital, especially of the Adult Infectious Disease ward and the Microbiology Laboratory.

Funding was provided by the Wellcome Trust (London, UK) and L'Institut de Recherche pour le Développement (Marseille, France).
Dr. Ming is an academic clinical fellow in infectious diseases at Imperial College London. His research interests include tropical medicine and parasitology.

\section{References}

1. Barratt J, Chan D, Sandaradura I, Malik R, Spielman D, Lee R, et al. Angiostrongylus cantonensis: a review of its distribution, molecular biology and clinical significance as a human pathogen. Parasitology. 2016;143:1087-118. http://dx.doi.org/10.1017/ S0031182016000652

2. Wang Q-PP, Lai D-HH, Zhu X-QQ, Chen X-GG, Lun Z-RR. Human angiostrongyliasis. Lancet Infect Dis. 2008;8:621-30. http://dx.doi.org/10.1016/S1473-3099(08)70229-9

3. Punyagupta S, Juttijudata P, Bunnag T. Eosinophilic meningitis in Thailand. Clinical studies of 484 typical cases probably caused by Angiostrongylus cantonensis. Am J Trop Med Hyg. 1975;24: 921-31. http://dx.doi.org/10.4269/ajtmh.1975.24.921

4. Morassutti AL, Rascoe LN, Handali S, DA Silva AJ, Wilkins PP, Graeff-Teixeira C. Cross-reactivity of the $31 \mathrm{kDa}$ antigen of Angiostrongylus cantonensis - dealing with the immunodiagnosis of meningoencephalitis. Parasitology. 2017;144:459-63. http://dx.doi.org/10.1017/S0031182016001918

5. Slom TJ, Cortese MM, Gerber SI, Jones RC, Holtz TH, Lopez AS, et al. An outbreak of eosinophilic meningitis caused by Angiostrongylus cantonensis in travelers returning from the Caribbean. N Engl J Med. 2002;346:668-75. http://dx.doi.org/ 10.1056/NEJMoa012462

6. Rodpai R, Intapan PM, Thanchomnang T, Sanpool O, Sadaow L, Laymanivong S, et al. Angiostrongylus cantonensis and A. malaysiensis broadly overlap in Thailand, Lao PDR, Cambodia and Myanmar: a molecular survey of larvae in land snails. PLoS One. 2016;11:e0161128. http://dx.doi.org/10.1371/ journal.pone. 0161128

7. Qvarnstrom Y, Sullivan JJ, Bishop HS, Hollingsworth R, da Silva AJ. PCR-based detection of Angiostrongylus cantonensis in tissue and mucus secretions from molluscan hosts. Appl Environ Microbiol. 2007;73:1415-9. http://dx.doi.org/10.1128/ AEM.01968-06

8. Qvarnstrom Y, Xayavong M, da Silva AC, Park SY, Whelen AC, Calimlim PS, et al. Real-time polymerase chain reaction detection of Angiostrongylus cantonensis DNA in cerebrospinal fluid from patients with eosinophilic meningitis. Am J Trop Med Hyg. 2016;94:176-81. http://dx.doi.org/10.4269/ajtmh.15-0146 
9. McBride A, Chau TTH, Hong NTT, Mai NTH, Anh NT, Thanh TT, et al. Angiostrongylus cantonensis is an important cause of eosinophilic meningitis in southern Vietnam. Clin Infect Dis. 2017;64:1784-7. http://dx.doi.org/10.1093/cid/cix118

Address for correspondence: Damien K.Y. Ming, Imperial College London, The Section of Infectious Diseases and Immunity, Commonwealth Building, Hammersmith Campus, London SW7 2AZ, UK; email: damien.ming@doctors.org.uk

\section{Tool for Eliminating Dog-Mediated Human Rabies through Mass Dog Vaccination Campaigns}

\author{
Eduardo A. Undurraga, ${ }^{1}$ Jesse D. Blanton, \\ S.M. Thumbi, Athman Mwatondo, \\ Mathew Muturi, Ryan M. Wallace
}

Author affiliations: Centers for Disease Control and Prevention, Atlanta, Georgia, USA (E.A. Undurraga, J.D. Blanton, R.M. Wallace); Kenya Medical Research Institute, Nairobi, Kenya (S.M. Thumbi); Washington State University, Pullman, Washington, USA (S.M. Thumbi); Kenya Ministry of Health, Nairobi (A. Mwatondo, M. Muturi)

DOI: https://doi.org/10.3201/eid2312.171148

The World Health Organization and collaborating agencies have set the goal of eliminating dog-mediated human rabies by 2030. Building on experience with rabies endemic countries, we constructed a user-friendly tool to help public health officials plan the resources needed to achieve this goal through mass vaccination of dogs.

$\mathrm{G}$ lobally, rabies kills $\approx 60,000$ persons annually; most $(\approx 99 \%)$ cases are transmitted by domestic dogs $(1-3)$. Controlling dog rabies through periodic mass vaccination campaigns substantially reduces human exposures (4). The elimination of dog rabies in most of the Western Hemisphere and countries in Asia has demonstrated the effectiveness and sustainability of vaccinating dogs $(5,6)$ by combining massive dog rabies vaccination with coordinated efforts of the medical and veterinary sectors (One Health approach), including education about responsible

${ }^{1}$ Current affiliation: Pontificia Universidad Católica de Chile,

Santiago, Chile. pet ownership, rabies awareness campaigns, and access to postexposure prophylaxis $(5,6)$.

The World Health Organization recommends that at least $70 \%$ of the dog population be vaccinated to control and potentially eliminate dog rabies (3). In 2016, WHO and partner organizations set the goal of eliminating dog-mediated human rabies by 2030 (7). This goal could be achieved by massive, costly administration of preexposure and postexposure prophylaxis, mass vaccination of dogs, or both.

Countries to which rabies is endemic in dogs are at different stages in their rabies control efforts $(5,8)$. Countries at early stages face barriers related to a limited understanding of the local epidemiology, logistic and operational challenges, competing priorities from other diseases, and lack of planning tools to reasonably project the resources needed. During 2016, we estimated the resources potentially required to eliminate dog rabies globally by 2030 (7). We combined multiple data sources to estimate 4 key factors that affect this goal: country development, cost of dog vaccination programs, potential demand for dog rabies vaccine, and estimated number of vaccinators. We aimed to realistically assess the global situation by highlighting the main challenges that might hamper elimination efforts. However, although these global estimates can inform an important discussion about global and regional strategic planning and resource mobilization, they are not necessarily useful to inform country-level decision-making. We addressed this limitation by providing a user-friendly tool that requires only limited country-specific data to help countries plan toward the goal of eliminating dog rabies through mass dog vaccination.

We designed the tool to plan for dog rabies elimination by 2030 (a 13-year framework). We assumed 13 years would be enough time for even the least developed rabies control programs to achieve elimination, provided the country was fully committed. The country's starting point within this time frame would depend on its current dog vaccination rate, and the given country would demonstrate incremental improvements in preparation for the vaccination campaign (e.g., training of workforce involved, dog population surveys) or in the proportion of the dog population vaccinated. The tool requires input of demographic data (human population, percentage urban, human-to-dog ratio); current dog vaccination coverage; logistic data for the campaigns (available vaccinators, dog vaccination rates, campaign duration); and an estimated cost per vaccinated dog. We constructed a worksheet to help users estimate the cost per vaccinated dog based on a pilot campaign. The tool (including assumptions, instructions, and data requirements) is available for public use (online Technical Appendix, https://wwwnc.cdc.gov/ EID/article/23/12/17-1148-Techapp1.xlsm) and already has been used in Haiti and Guatemala as part of a rabies elimination workshop held at the Centers for Disease Control and 